\title{
Effect of external stress on deuteride (hydride) precipitation in Zircaloy-4 using in situ neutron diffraction
}

\author{
Jun-li Lin ${ }^{\mathrm{a}}$, Ke An ${ }^{\mathrm{b}}$, Brent J. Heuser ${ }^{\mathrm{a}, *}$ \\ ${ }^{a}$ Department of Nuclear, Plasma, and Radiological Engineering, University of Illinois at Urbana-Champaign, Urbana, IL 61801, USA \\ ${ }^{b}$ Chemical and Engineering Materials Division, Oak Ridge National Laboratory, Oak Ridge, TN 37831, USA
}

\begin{abstract}
In situ neutron diffraction is utilized to study the deuteride (hydride) precipitation behavior in a cold-worked stress-relieved (CWSR) Zircaloy-4 material upon cooling from $420^{\circ} \mathrm{C}$ to room temperature with a $78 \mathrm{MPa}$ external stress applied along the rolling direction (RD) of the material. Two general methods, c-axis strain analysis and direct calculation from the diffraction-measured deuteride volume fraction, have been applied to study the deuterium concentration in the zirconium solid solution. The terminal solid solubility of precipitation (TSSP) for deuterium in the solution is then described using the Arrhenius equation. It is observed that the TSSP given by the c-strain analysis is in good agreement with the external stress-free data and the model predictions, while the TSSP given by the direct calculation from the deuteride volume fraction overestimates the amount of deuterium in the solution. The $78 \mathrm{MPa}$ external stress applied in this study is observed to have small effect on the deuterium solubility in the material.

Keywords: Neutron diffraction, Zirconium alloys, Zirconium deuteride (hydride), Deuterium (Hydrogen) solubility
\end{abstract}

*Corresponding author: Tel: +1-217-333-9610, email: bheuser@illinois.edu 


\section{Introduction}

Zircaloy is one of the most common fuel cladding materials used in light water reactors due to excellent mechanical properties, corrosion resistance and low thermal neutron absorption crosssection. During operation hydrogen is created by corrosion of outer wall of the cladding and radiolysis of the water coolant. A fraction of hydrogen diffuses into the cladding and is dissolved in the zirconium solid solution, and eventually precipitates out as zirconium hydrides when the hydrogen concentration exceeds the terminal solid solubility [1]. These hydrides distribute inhomogeneously within the cladding. The most typical observation of this inhomogeneity is the hydride rim (a near-surface layer contained high concentration of hydrides) and the hydride blister structure (a near-surface layer contained very high concentration of hydrides or bulky solid hydride phase) on the water-side of post-service cladding due to presence of the temperature gradient in the radial direction [2]. The hydrogen concentration in the rim structure can reach 1300 wppm while the average concentration of the cladding is approximate 400 wppm [3].

Besides the radial direction in the cladding, temperature gradients also exist in the axial and azimuthal directions and other components during the operation. This is caused by a combination effect of heat production in the fuel, local heat transfer conditions, local oxide growth and heterogeneity of the core geometry. Regions of colder temperature such as inter-pellet gaps, corners and side of assemblies have been observed to have higher hydride concentration $[4,5]$. This heterogeneous hydride distribution causes a significant loss of ductility of the cladding, while the brittle fracture characteristics of a hydride blister morphology has been observed occur at a very broad temperature range of 25 to $400{ }^{\circ} \mathrm{C}$ [6].

Different local stress states in the components can also affect the hydride distribution. It is known that precipitation of the less dense hydride phase ( $\delta$-hydride) will elastically and plasti- 
cally distort the zirconium phase [7]. This plastic strain energy is compensated by undercooling required to precipitate the hydride upon cooling. Hence the precipitation temperature (temperature to start precipitating the hydride also known as TSSP) of zirconium hydride is lower than the dissolution temperature (temperature to fully dissolve the hydride also known as TSSD), resulting in a hysteresis between the TSSD and TSSP solvi. In principal, the strain energy provided by an external tensile should reduce the hysteresis by decreasing the TSSD and increasing the TSSP solvus $[8,9]$.

Understanding of the effect of stress on the hydrogen solubility and hydride precipitation behavior in the zirconium alloys is critical for accurate prediction on the fuel performance, either during the service or in the post-service storage. For example, current delayed hydride cracking (DHC) model significantly relies on the hydride precipitation/dissolution behavior under a stressed state [10]. However, there are very limited experimental data that have studied the hydrogen solid solubility and the hydride precipitation under an applied load [11, 12]. This work studies the dependence of hydrogen terminal solid solubility (TSS) on the external tensile stress utilizes the in situ time of flight (TOF) neutron diffraction, with two stationary banks that record two different grain orientation families. The effect of the tensile stress is examined by comparing the data in this work with other stress-free experimental results and model predictions.

\section{Experiment}

\subsection{Sample preparation}

Cold-worked stress-relieved (CWSR) Zircaloy-4 material with the thickness of $3 \mathrm{~mm}$ was provided by ATI Specialty Alloys and Components. This relative thick sample is used to increase the measured volume and the neutron diffraction signal. Pole figure analysis of this material shows a typical basal plane orientation of the CWSR Zircaloy [1, 13], with the alignment of two 
basal poles tilted $30-40^{\circ}$ from the normal direction (ND) and resides within the RD-TD plane. The chemical composition of the plate is consistent with ASTM Standards B353-12 specification for Zircaloy-4 [14].

Dog-bone tensile specimens were machined using the electrical discharge machining (EDM) to avoid generating the machining residual stress. The dimension of this tensile specimen is specified in Fig. 1b. The surfaces of the tensile specimens were mechanically polished to 0.05 $\mu \mathrm{m}$ silica suspension. A thin film of $\mathrm{Ni}(\sim 0.2 \mu \mathrm{m})$ was sputter coated on the polished surface to enhance the near-surface deuterium diffusion into the specimen $[6,13]$.

The specimen was charged with deuterium using a Sievert-type apparatus gas system [15]. Deuterium is selected over hydrogen in this experiment due to its low neutron incoherent scattering cross section and large coherent scattering cross section. The deuterium charging was performed at $400{ }^{\circ} \mathrm{C}$ under the stress-free condition in a vacuum $\left(10^{-8}\right.$ torr $)$ environment. The variation of the deuterium partial pressure in the system is monitored by capacitance manometer gauges until the desired amount of deuterium concentration in the specimen has been reached [15]. Specimens were then slowly cooled $\left(1^{\circ} \mathrm{C} \mathrm{min}^{-1}\right)$ to the room temperature to ensure that only the $\delta$-deuteride precipitated [16]. This gas charging method aims to create a thin layer of deuteride rim/blister near the surface of the specimen to simulate the hydride rim structure observed on the post-service cladding tubes. The microscopic examination shown on Fig. 1a from the ND-RD plane of the specimen shows a $\sim 20 \mu \mathrm{m}$ near-surface deuteride rim/blister structure with an average deuterium concentration of $1054 \mathrm{wppm}$ throughout the sample. This is consistent with the observation by Lin et al. [13] in terms of the hydrogen-equivalent concentration (527 wppm of hydrogen in this study versus $500 \mathrm{wppm}$ of hydrogen in [13]). 


\subsection{Time-of-flight neutron diffraction and data analysis}

In situ time-of-flight (TOF) neutron diffraction experiment was conducted using the VULCAN engineering materials diffractometer at Spallation Neutron Source (SNS) at Oak Ridge National Laboratory [17]. The VULCAN-MTS load frame system and an induction coil were used for in situ mechanical and thermal testing. The sample was heated to $400{ }^{\circ} \mathrm{C}$, held for 1.5 hours then cooled $1{ }^{\circ} \mathrm{C} \mathrm{min}-1$ to the room temperature with an $78 \mathrm{MPa}$ constant external stress applied along the RD. TOF neutron diffraction spectra was continuously recorded throughout the experiment using the two stationary bank detectors at the diffraction angles of $\pm 90^{\circ}$. The in situ experimental procedure and the arrangement are schematically shown in Fig. 1. This study also demonstrates the ability of VULCAN to perform an in situ study on a minor phase in a bulk material, such as the zirconium deuteride phase in this work.

The TOF neutron diffraction spectra are chopped and binned into 8 increments with each increment contains 60 mins of the neutron exposure time. The temperature and stress magnitude of each increment are therefore an average value in the corresponding 60 mins period. This relative long exposure time is necessary to obtain the statistically significant minor phase peaks from the zirconium deuteride . Data analysis (spectra integration and peak fitting) is performed using VULCAN Data Reduction and Interactive Visualization (VDRIVE) software to obtain the diffraction peak information for the two phases ( $\alpha$-Zr and $\delta$-deuteride), such as variation of the peak intensity and plane spacing throughout the in situ measurement.

The binned spectra were first normalized to the incoherent vanadium diffraction spectra and the proton charge. The Debye-Waller factors and the change of texture is expected to be negligible throughout the experiment [18]. Thus, it is plausible to assume that any change of the peak intensity is caused by the change of the amount of deuteride in the measured volume, and the volume fraction of the $\delta$-deuteride phase in the measured volume is linearly proportional to 
the measured integrated peak intensity $[11,18]$. Therefore, the calculation of the amount of deuterium in the $\delta$-deuteride phase can be based on the evolution of the $\delta$-(220) reflection intensity since this peak is not overlapped by the strong zirconium peaks, as can be seen in Fig. 2. The $\delta$-(220) provides the best single peak fit result in this study. Although the $\delta$-(311) has higher multiplicity factor (24) than the $\delta$-(220) reflection (12), the observed counting statistic is much worse than the $\delta$-(220) in this case. The integrated $\delta$-(220) peak intensity measured at $70{ }^{\circ} \mathrm{C}$ is used as a reference with deuterium fully precipitated, corresponding to the deuteride volume fraction deduced from the known average deuterium concentration in the specimen (1054 wppm deuterium). We note that the solubility of deuterium in the zirconium at $70{ }^{\circ} \mathrm{C}$ is approximated 2 wppm [19], thus taking $70^{\circ} \mathrm{C}$ as the reference will slightly overestimate the amount of deuterium in the deuteride phase, but this deviation is negligible.

Using the aforementioned linear relationship between the $\delta$-deuteride volume fraction and the measured integrated peak intensity, the evolution of the deuteride phase volume fraction throughout cooling can be expressed as:

$$
V_{f}^{\delta}(T)=V_{f, 0}^{\delta} \times \frac{I_{(220)}^{\delta}(T)}{I_{(220), 0}^{\delta}}
$$

where $V_{f}^{\delta}(T)$ is the volume fraction of the $\delta$-phase at temperature $T, V_{f, 0}^{\delta}$ is the volume fraction of the $\delta$-phase when deuteride is fully precipitated (the $70{ }^{\circ} \mathrm{C}$ reference), $I_{(220)}^{\delta}(T)$ and $I_{(220), 0}^{\delta}$ are the measured integrated $\delta$-(220) peak intensity at the temperature $T$ and $70{ }^{\circ} \mathrm{C}$, respectively. The amount of deuterium in the Zircaloy solid solution is then determined by applying the lever rule in a $\mathrm{Zr}-\mathrm{H}$ phase diagram [20]:

$$
[D]_{\alpha}=\frac{[D]_{\mathrm{tot}}-w_{f}^{\delta}[D]^{\delta}}{1-w_{f}^{\delta}}
$$

where $[D]_{\alpha}$ and $[D]_{\text {tot }}$ is the equilibrium amount of deuterium in the solid solution and the average amount of deuterium in the sample, respectively, $w_{f}^{\delta}$ is the weight fraction of the $\delta$-phase, and 
$[D]_{\delta}$ is the equilibrium deuterium concentration in the $\delta$-phase. $w_{f}^{\delta}$ is simply the conversion of the measured $V_{f}^{\delta}$ in Eq. (1), using the density values of the two phases in [21]. The $\delta$-phase boundary measured in the $\mathrm{Zr}-\mathrm{H}$ binary phase diagram [20] is used to calculate $[D]^{\delta}$ as a function of temperature, assuming that the $\delta$-hydride and the $\delta$-deuteride has the same stoichiometry. A similar method was used in [18].

\section{Results}

The $\delta(111)$ and $\delta(220)$ reflections recorded in Bank 2 detector (ND) are shown in Fig. 2. It is clear the $\delta(220)$ reflection has the best counting statistic and provides the most reliable single peak fit result. $\delta(311)$ has poor counting statistic and is not shown. It is also worth noting here that the $\delta$ is the only deuteride phase observed in this study, which is a common observation in CWSR Zry-4 material with a similar hydrogen-equivalent concentration and has precipitated hydride in a slow cooling rate $\left(1^{\circ} \mathrm{min}^{-1}\right)$ [22]. No reflections from other deuteride phases $(\gamma$ and $\varepsilon)$ were observed.

Fig. 3a shows the evolution of the deuterium concentration in the zirconium solid solution throughout the experiment, determined from the variation of the normalized $\delta(220)$ peak area then calculated using Eq. (2). Evolution of the deuterium concentration in the solid solution as a function of temperature can then be fitted using an Arrhenius equation to quantify the TSSP line:

$$
C_{\mathrm{TSSP}}=A \exp \left(-Q_{p} / R T\right)
$$

where $C_{\text {TSSP }}$ is the equilibrium deuterium solvus upon cooling. $A$ is a pre-exponential constant and $Q_{p}$ is the precipitation activation energy which includes, in our case, the change of enthalpy for deuterium in solid solution, the strain energy to accommodate the precipitation of less dense deuteride and the work done by the external stress. $Q_{p}$ will be discussed more in the Discussion 
section.

The Arrhenius fit is shown in Fig. 3b, using the York linear regression method [23] which weights in the uncertainty that is propagated from the diffraction peak fitting. The TSSP solvus in the two directions are listed below :

$$
\begin{aligned}
& C_{D}^{N D}=350850 \exp \left(\frac{-20151}{R T}\right) \\
& C_{D}^{R D}=261330 \exp \left(\frac{-18325}{R T}\right)
\end{aligned}
$$

where $C_{D}$ is the measured equilibrium deuterium solvus with superscripts that denotes the corresponding direction.

\section{Discussion}

Before further interpretation, we first note that the previous studies have shown that the TSSD is a fairly stable value but the TSSP could be significantly affected by the thermal history for hydrogen in zirconium solid solution [19]. Examples of the relevant thermal history are heating/cooling rate for dissolving/precipitating the deuteride (hydride), maximum temperature during the heating and holding period at the maximum temperature [24]. The composition (only minor difference exist between Zircaloy families) and the microstructure of the material has little effect on both the TSSD and the TSSP [24]. Table 1 summarizes the experimentally measured TSSP solvus of our work and other studies under the stated experimental conditions.

We observed that, based on the experimental conditions and techniques applied, the externally applied stress decreases the activation energy $\left(Q_{p}\right)$ for hydride/deuteride precipitation. In this work with $78 \mathrm{MPa}$ stress was applied along the $\mathrm{RD}, Q_{p}$ is decreased by $\sim 11 \mathrm{~kJ} / \mathrm{mol}$, when compared with the external stress-free TSSP solvus. Other work done by Colas et al. [11] with $160 \mathrm{MPa}$ stress applied along the TD, $Q_{p}$ was decreased by $\sim 18 \mathrm{~kJ} / \mathrm{mol}$. These results imply 
the higher the applied stress is, the lower the $Q_{p}$ will be. The theoretical model for the effect of the elastic stress on the equilibrium solvus of dissolved hydrogen in the metal solid solution was developed as [27]:

$$
C_{H}^{\sigma_{h}}=C_{H}^{0} \exp \left(\frac{\bar{V}_{H} \sigma_{h}}{R T}\right)
$$

where $C_{H}^{\sigma_{h}}$ is the equilibrium hydrogen solvus for a measured volume under a hydrostatic stress $\sigma_{h}$ and $C_{H}^{0}$ is the equilibrium hydrogen solvus for a region where the $\sigma_{h}=0 . \bar{V}_{H}$ is the partial molar volume of hydrogen in the metal and $R T$ have their usual meaning. Eqn. (5) was further modified by Coleman and Ambler [28] by assuming the precipitation of hydride phase and the dissolved hydrogen were in equilibrium, the solvus then becomes:

$$
C_{H}^{\sigma_{h}}=C_{H}^{0} \exp \left(\frac{\left(\bar{V}_{H}^{h}-\bar{V}_{H}\right) \sigma_{h}}{R T}\right)
$$

where $\bar{V}_{H}^{h}$ is the hydrogen partial molar volume associated with the precipitation of hydride. A similar equation was also developed by Puls [8, 29]:

$$
C_{H}^{\sigma_{h}}=C_{H}^{0} \exp \frac{\bar{W}^{\mathrm{a}}}{x R T} \exp \frac{\sigma_{h} \bar{V}_{H}}{R T}
$$

\begin{tabular}{|c|c|c|c|c|c|c|c|}
\hline \multirow[t]{2}{*}{ Author } & \multirow{2}{*}{$\begin{array}{c}{[H]_{\text {tot }}} \\
\text { (wppm) }\end{array}$} & \multirow{2}{*}{$\begin{array}{l}T_{\max } \\
\left({ }^{\circ} \mathrm{C}\right)\end{array}$} & \multirow{2}{*}{$\begin{array}{l}\text { heating/cooling rate } \\
\qquad\left({ }^{\circ} \mathrm{C} / \mathrm{min}\right)\end{array}$} & \multirow{2}{*}{$\begin{array}{l}\text { holding time } \\
\qquad(\min )\end{array}$} & \multirow{2}{*}{$\begin{array}{l}\sigma_{\text {applied }} \\
(\mathrm{MPa})\end{array}$} & \multicolumn{2}{|c|}{ TSSP solvus } \\
\hline & & & & & & $A$ (wppm) & $Q_{p}(\mathrm{~J} / \mathrm{mol})$ \\
\hline This work (RD) & 527 (H-equivalent) & 400 & $25 / 1$ & 60 & $78 / / \mathrm{RD}$ & 18200 & $20295 \pm 1426$ \\
\hline This work (ND) & 527 (H-equivalent) & 400 & $25 / 1$ & 60 & $78 / / \mathrm{RD}$ & 13744 & $18545 \pm 1238$ \\
\hline This work from c-strain & 527 (H-equivalent) & 400 & $25 / 1$ & 60 & $78 / / \mathrm{RD}$ & 11181 & $19313 \pm 349$ \\
\hline McMinn 2000 [24] & 20 to 80 & $\sim 330$ & $2 / 2$ & None & None & 138746 & 34467.52 \\
\hline Tang 2009 [25] & 20 to 240 & 500 & $10 / 10$ & 5 & None & 40135 & 27336 \\
\hline Zanellato 2012 [18] & 475 to 600 & 570 & $5 / 5$ & 30 & None & 66440 & $29630 \pm 160$ \\
\hline Colas 2013 [11] & 190 to 350 & 450 & $25 / 1$ & $\sim 60$ & $160 / / \mathrm{TD}$ & & 12040 \\
\hline Kim 2014 [26] & 40 to 731 & 550 & $20 / 20$ & 5 & None & 47220 & 26843 \\
\hline
\end{tabular}

Table 1: Summary of the TSSP experimental conditions and results for hydride/deuteride in Zry-4 materials. 
where $x$ is the number of mole of hydrogen in a mole of hydride $\mathrm{ZrH}_{x}$. The total molar accommodation energy $\bar{W}^{\mathrm{a}}$, is the energy associated with the accommodation of the hydride phase volume change in an external stress field, and is given by $\bar{W}^{\mathrm{a}}=-\bar{V}_{H}^{h} \sigma_{i j} \epsilon_{i j}^{\text {,hyd }}$ where $\epsilon_{i j}^{\text {hyd }}$ is the stress-free strain transformed from the zirconium to the hydride phase.

Carpenter [7] has estimated $\epsilon_{i j}^{\text {hyd }}$ theoretically using the misfit between lattice cells of the two phases ( $\delta$-hydride and $\alpha$-Zr) with a specific orientation relationship of $\delta(111) \| \alpha(0001)$. The result was then further extended by Singh et al. [30] by including a temperature dependence. $\bar{V}_{H}$ and $\bar{V}_{H}^{h}$ were measured in [31], and the difference $\left(\bar{V}_{H}^{h}-\bar{V}_{H}\right)$ is at most $-0.27 \mathrm{~cm}^{3}$ per mole of hydrogen. Considering the texture of the Zry-4 studied in this work where the most of basal plane normals reside within the ND-TD plane but tilted closer to the ND, we assume that the ND corresponds to the basal plane normal (0001) direction, while the RD and the TD corresponds to the primary $(10 \overline{1} 0)$ and the secondary $(11 \overline{2} 0)$ prismatic plane normal directions in Carpenter's calculation, respectively. $\epsilon_{i j}^{\text {hyd }}$ from [30] is then re-written in a matrix form:

$$
\epsilon_{i j}^{\text {hyd }}=\left[\begin{array}{ccc}
0.0371+2.3111 \times 10^{-5} T & 0 & 0 \\
0 & 0.0371+2.311 \times 10^{-5} T & 0 \\
0 & 0 & 0.06463+1.9315 \times 10^{-5} T
\end{array}\right]
$$

where $T$ is the temperature in K. $\epsilon_{11}^{\text {hyd }}, \epsilon_{22}^{\text {hyd }}$ and $\epsilon_{33}^{\text {hyd }}$ corresponds to the RD, TD and ND, respectively.

Considering the magnitude of external stress applied in this study and by Colas et al. [11], with the $x$ obtained from the $\delta$-phase boundary line in the $\mathrm{Zr}$ - $\mathrm{H}$ phase diagram [20], both models (Eqn. (6) and (7)) predict that the change of $Q_{p}$ due to the external stress is less than $0.1 \mathrm{~kJ} / \mathrm{mol}$ and the effect on the solubility is very limited. This is inconsistent with the experimental observation both in this work and in Colas et al. [11]. Similar results are obtained if the form for $\epsilon_{i j}^{\text {hyd }}$ proposed by Perovic et al. [32] is used, where the $\delta$ precipitation is assumed as an invariant plane 
martensitic transformation.

In fact, in a work by Puls [33], Eqn. (7) was numerically evaluated at the crack tip area which can be considered as an extreme case since the hydrostatic stress can approach $1800 \mathrm{MPa}$. However, even at high stress $Q_{p}$ is only reduced by an order of $0.1 \mathrm{~kJ} / \mathrm{mole}$. This discrepancy between the models and the experimental results is shown in Fig. 4a, in a form of the hydrogenequivalent TSSP solvus line as the function of temperature. In Fig. 4a the TSSP in this work is shifted to lower temperature and the TSSP by Colas et al. [11] is shifted to higher temperature, with respect to the external stress-free solvus. The predicted TSSP with $78 \mathrm{MPa}$ applied stress is, however, almost the same as the external stress-free solvus. The result in Fig. 4a also suggests that although $Q_{P}$ in this work and in [11] were both decreased by the external stress, the TSSPs were shifted to different directions. It should be noted that the external stress was applied along the RD and the TD in this work and in [11], respectively. This observation is further discussed in the following section.

\subsection{Effect of applied stress on deuteride precipitation and deuterium solubility}

The observed discrepancy between the model and experiments is attributed to external stress affecting differently on the hydride precipitation in different grain sets. In other words, the hydride precipitation behavior and hydrogen solubility responses differently between grains with different crystallography orientations. A study done by Vizcaíno et al. [12] observed that the external stress increased the amount of hydride precipitation within the grains which have their basal plane normals parallel to the applied stress, while the amount of hydrogen in the solid solution in the same group of grains was not affected. This also implies, because of the mass conservation, that the redistribution of hydride particles occur while the external stress was applied. The hydride redistribution under a stress is also commonly known as the root cause of the 
delayed hydride cracking (DHC), as the applied stress increases the chemical potential in a local area (i.e., at the crack tip) which creates a hydrogen flux that flows toward to the stressed area [33].

Since the effect of the external stress on the hydride precipitation is restricted to certain grains, one can expect that for techniques which measure the hydride dissolution and precipitation response using the bulk average method (taking into account of all crystallography orientations), such as using differential scanning calorimetry, internal friction, elastic modulus or integrating a whole diffraction ring [12], this effect will not be detected. On the other hand, for techniques or analysis methods which investigate the hydride behavior in a group of grains with a similar orientation under the applied stress, it may lead to different results by calculating the amount of hydrogen in the solid solution from the amount of hydrogen as the hydride. Examples of the latter case are neutron diffraction applied in this study which uses two bank detectors (Fig. 1a) and the synchrotron diffraction work by Colas et al. [11] with intensity spanned $\pm 10^{\circ}$ along the loading axis. Both works used the detected hydride volume fraction to calculate the amount of hydrogen in the matrix solid solution (Eqn. (2) in this study and Eqn. (3) in [11]). This could explain the disagreement between the experimental works and the models.

Another method to measure the amount of hydrogen in the solid solution without reference to the diffraction-detected hydride fraction is to use the known dependency of the zirconium lattice parameter on the amount of dissolved hydrogen. This was done in [12]. The dilatation of the zirconium lattice parameter due to the hydrogen in the interstitial sites is anisotropic with the larger strain observed along the c-axis [31]. Thus, the c-axis strain is more sensitive to the variation of hydrogen concentration in the solid solution. The value of this dilatation was measured as $\frac{1}{c_{0}} \frac{d c}{d C_{D}} \simeq 5.415 \mu \varepsilon$ per wppm of deuterium in the zirconium solid solution with the negligible temperature dependence [31]. 
The evolution of the zirconium c-axis strain throughout this experiment is calculated using the d-spacing of the $\alpha(0002)$ basal plane. The thermal expansion strain [34] and the elastic strain [35] induced by the external stress on the c-axis is subtracted before we evaluated the strain induced by the deuterium in the solution. This analysis is only applicable in the ND (bank 2 detector in this case), since the $\alpha(0002)$ peak was absent in the RD due to the strong texture of the study material.

The evolution of the c-axis lattice parameter as a function of temperature is shown in Fig. 5a along with the predicted thermal expansion value. Fig. 5b shows c-axis strain after subtraction of the thermal and the mechanical induced strain. Equation 2, which refers the amount of deuterium in the solid solution from the direct diffraction measurement on the deuteride phase, overestimates the deuterium solubility in the matrix as shown in Fig. 5c. This agrees with the statement that hydride particles tend to redistribute and accumulate in a high tensile stress area.

In this experiment the matrix grains with their basal normal parallel to the ND were compressed, this decreases the deuteride fraction $\left(w_{f}^{\delta}\right.$ in Eqn. (2)) in these grains and increases the calculated amount of deuterium in the solution. Likewise for Colas et al. [11], the applied 160 MPa tensile stress along the TD increases the amount of hydride particles in this direction and underestimates the amount of dissolved hydrogen in the solid solution (see Fig. 4a).

It was expected that if the deuteride re-distribution occurred in our study, the solvus in the RD would be decreased and should be lower than the external stress free solvus (the red solid line in Fig. 4). This is because the deuteride volume fraction in the RD would increase to compensate the decreased amount in the ND. However, this phenomenon was not observed as Fig. 3a shows that the TSSP solvus in the ND and RD track together. As pointed out by Hardie [36], hydride reorientation is favored in grains with basal poles parallel to the direction of the applied stress, namely, grains in the ND and the TD for the CWSR Zry-4 material. In other words, hydride 
reorientation will be difficult to occur in the grain set along the $\mathrm{RD}$ of the material. It is possible that deuteride phase re-distributed and re-precipitated in other grain sets which could not be detected due to the limited angle covered by the two stationary detectors.

The Arrhenius equation is again be applied to fit the deuterium concentration in the solution obtained using the c-axis strain. The fitted TSSP solvus, denoted with a superscript c-strain, is:

$$
C_{\mathrm{D}}^{\text {c-strain }}=11181 \exp \left(\frac{-19313}{R T}\right)
$$

and is also listed in Table 1. Although the precipitation activation energy $\left(Q_{p}\right)$ between the two obtained TSSP solvus (Eqn. (4) and (9)) using the two methods are similar, the difference can be observed in Fig. 4b. The stressed solvus (with $78 \mathrm{MPa}$ along the RD), obtained using the zirconium c-axis strain (Eqn. (9)), is now in good agreement with an external stress free TSSP solvus which is the average solvus of several literature results (values listed in Table 1). This result indicates that the external applied stress only slightly affects the hydrogen/dueterium solubility in the zirconium solid solution.

Finally, the relatively low precipitation activation energy $Q_{p}$ observed in this study could refer to the additional strain along the c-axis induced by the deuteride formation. This strain is anisotropic and can be as high as 0.072 along the c-axis ( $\epsilon_{i j}^{\text {hyd }}$ in the last section). Presumably the strain is linearly proportional to the deuteride volume fraction and this additional strain will mostly affect the c-axis strain in the low temperature regime where the deuteride phase has been fully precipitated. Since the amount of deuterium in the solid solution is calculated from the $\mathrm{c}$-axis strain, subtracting the strain induced by the deuteride formation from the c-axis strain will decrease the amount of dissolved deuterium in the low temperature regime. This will increase the slope in the Arrhenius plot and increase the $Q_{p}$. Again considering the uncertainties in the reported literature values and this work, it is reasonable to conclude that the applied stress only 
slightly affects the $Q_{p}$ of deuteride in the Zry-4 material.

\section{Conclusion}

The work studies the effect of the external stress on the deuteride precipitation behavior in a CWSR Zry-4 material with the main observations are summarized below:

1. $\delta$-zirconium deuteride is the only deuteride phase observed by neutron diffraction upon a $1^{\circ} \mathrm{C} \mathrm{min}^{-1}$ cooling rate under the deuterium concentration applied in this study.

2. Two general methods, which are c-strain analysis and direct calculation from the diffractionmeasured deuteride volume fraction, were applied to study the deuterium concentration in a CWSR Zry-4 sample. The TSSP solvus obtained from the c-strain analysis is in good agreement with the experimental external stress-free solvus from previous literature results and model prediction, while the TSSP calculated from the measured deuteride phase fraction is shifted to a lower temperature.

3. Comparing results from previous literatures, model prediction and this work, we believe the c-strain analysis provides more accurate measurement on the deuterium/hydrogen solvus when an external stress is applied. This is because the external stress causes re-distribution of the deuteride/hydride phase among different grain sets which disturbs the hydrogen/deuterium concentration measurement using the detected hydride/deuteride phase fraction.

4. Application of a $78 \mathrm{MPa}$ tensile stress along the RD upon cooling does not change the deuterium TSSP solvus in the CWSR Zry-4 material. 


\section{Acknowledgments}

The authors greatly appreciate access to the VULCAN instrument. The authors would also like to acknowledge A. Stoica and M. Frost for their assistance with the neutron experimental work at VULCAN. This work was supported by the US Department of Energy Nuclear Energy Fuel Aging in Storage and Transportation under Grant No. IRP-2011-05352, and by the US Department of Energy Nuclear Energy University Programs Integrated Research Project under contract number IRP-12-4728. The work was also carried out in part in the Frederick Seitz Materials Research Laboratory Central Research Facilities, University of Illinois. This research used resources at the Spallation Neutron Source, a DOE Office of Science User Facility operated by the Oak Ridge National Laboratory. 


\section{References}

[1] C. Lemaignan, A. T. Motta, Materials Science and Technology (1994).

[2] A. Sawatzky, Canadian Metallurgical Quarterly 24 (1985) 227-234.

[3] P. Bossis, D. Pecheur, K. Hanifi, J. Thomazet, M. Blat, in: 14th International Symposium on Zirconium in the Nuclear Industry, volume 3, pp. 494-525.

[4] A. M. Garde, S. R. Pati, M. A. Krammen, G. P. Smith, R. K. Endter, in: Zirconium in the Nuclear Industry: Tenth International Symposium, ASTM International.

[5] O. Courty, A. T. Motta, J. D. Hales, Journal of Nuclear Materials 452 (2014) 311-320.

[6] O. Pierron, D. Koss, A. Motta, K. Chan, Journal of nuclear materials 322 (2003) 21-35.

[7] G. Carpenter, Journal of Nuclear Materials 48 (1973) 264-266.

[8] M. Puls, Acta Metallurgica 29 (1981) 1961-1968.

[9] T. B. Flannagan, N. Mason, H. Birnbaum, The Effect of Stress on Hydride Precipitation., Technical Report, DTIC Document, 1980

[10] M. Puls, Metallurgical Transactions A 21 (1990) 2905-2917.

[11] K. B. Colas, A. T. Motta, M. R. Daymond, J. D. Almer, Journal of Nuclear Materials 440 (2013) 586-595.

[12] P. Vizcaíno, J. Santisteban, M. V. Alvarez, A. Banchik, J. Almer, Journal of Nuclear Materials 447 (2014) 82-93.

[13] J.-1. Lin, X. Han, B. J. Heuser, J. D. Almer, Journal of Nuclear Materials 471 (2016) 299-307.

[14] Astm standard b353-12, specification for wrought zirconium and zirconium alloy seamless and welded tubes for nuclear service (except nuclear fuel cladding), 2012. URL: www . astm. org. doi:10.1520/B0353-12.

[15] W. Chen, B. J. Heuser, Journal of alloys and compounds 312 (2000) 176-180.

[16] J. Bradbrook, G. Lorimer, N. Ridley, Journal of Nuclear Materials 42 (1972) 142-160.

[17] X.-L. Wang, T. Holden, G. Q. Rennich, A. Stoica, P. K. Liaw, H. Choo, C. R. Hubbard, Physica B: Condensed Matter 385 (2006) 673-675.

[18] O. Zanellato, M. Preuss, J.-Y. Buffiere, F. Ribeiro, A. Steuwer, J. Desquines, J. Andrieux, B. Krebs, Journal of Nuclear Materials 420 (2012) 537-547.

[19] Z. Pan, I. Ritchie, M. Puls, Journal of nuclear materials 228 (1996) 227-237.

[20] E. Zuzek, J. Abriata, A. San-Martin, F. Manchester, Bulletin of alloy phase diagrams 11 (1990) 385-395.

[21] S. Yamanaka, K. Yamada, K. Kurosaki, M. Uno, K. Takeda, H. Anada, T. Matsuda, S. Kobayashi, Journal of alloys and compounds 330 (2002) 99-104. 
[22] R. Daum, Y. Chu, A. Motta, Journal of Nuclear Materials 392 (2009) 453-463.

[23] D. York, N. M. Evensen, M. L. Martınez, J. D. B. Delgado, American Journal of Physics 72 (2004) $367-375$.

[24] A. McMinn, E. C. Darby, J. S. Schofield, in: Zirconium in the Nuclear Industry: Twelfth International Symposium, ASTM International

[25] R. Tang, X. Yang, International Journal of Hydrogen Energy 34 (2009) 7269-7274.

[26] J.-S. Kim, Y.-S. Kim, International Journal of Hydrogen Energy 39 (2014) 16442-16449.

[27] J. Li, R. Oriani, L. Darken, Zeitschrift für Physikalische Chemie 49 (1966) 271-290.

[28] C. Coleman, J. Ambler, Scripta Metallurgica 17 (1983) 77-82.

[29] M. Puls, Acta Metallurgica 32 (1984) 1259-1269.

[30] R. N. Singh, P. Ståhle, A. R. Massih, A. Shmakov, Journal of Alloys and Compounds 436 (2007) 150-154.

[31] S. MacEwen, C. Coleman, C. Ells, J. Faber, Acta Metallurgica 33 (1985) 753-757.

[32] V. Perovic, G. Weatherly, C. Simpson, Acta Metallurgica 31 (1983) 1381-1391.

[33] M. P. Puls, Journal of nuclear materials 393 (2009) 350-367.

[34] J. Goldak, L. Lloyd, C. Barrett, Physical Review 144 (1966) 478.

[35] E. Fisher, C. Renken, Physical Review 135 (1964) A482.

[36] D. Hardie, M. Shanahan, Journal of Nuclear Materials 55 (1975) 1-13. 


\section{List of Figures}

1 (a) Experimental arrangement of VULCAN. Bank 1 and Bank 2 recorded the lattice planes with their normal parallel to Q1 and Q2, respectively. Q1 and Q2 correspond to the RD and ND in terms of the sample orientation, respectively. The external load is applied along the RD, while the deuteride rim/blister is on the ND surface. The incident neutron beam is at $45^{\circ}$ with respect to the ND. (b) The dimension of the dog-bone tensile specimen. (c) The thermal and mechanical history throughout the in situ experiment. . . . . . . . . . . . .

2 Examples of partial neutron diffraction spectrums from the (a) $\delta(220)$ reflection and the (b) $\delta(111)$ reflection. The red solid line represents the spectrum taken at $400{ }^{\circ} \mathrm{C}$ while the black solid line was taken at $70{ }^{\circ} \mathrm{C}$.

3 (a) Evolution of the deuterium concentration in the zirconium solid solution in the unit of wppm. The uncertainties are within the size of the data points. A subplot shows the corresponding temperature and stress at each data points. (b) Arrhenius fit on the evolution of deuterium concentration in the zirconium solid solution. . . . . . . . . . . . . . . . .

4 (a) TSSP of hydrogen in the Zry-4 solid solution, showing the discrepancy between the experimental observation and theoretical calculation for the effect of the external stress on the solvus. Blue lines represent the experimental measurements in this work along the two directions with $78 \mathrm{MPa}$ of tensile stress applied along the RD. Red line is an average solvus of several literature values that are listed in Table. 1, also serves as the zero external stress solvus for the theoretical models (Eqn. (6) and (7)). Black line is a theoretically calculated solvus based on the red line with a $78 \mathrm{MPa}$ uniaxial stress applied. (b) Same as (a) but including the solvus calculated using the c-axis dilatation in this work represented as a black line and excluding the theoretically calculated solvus for clearance . . . . .

5 (a) The evolution of the c lattice parameter as function of the temperature in this study along with the predicted thermal expansion line displayed in a red dash line. (b) The strain on the c-axis after subtraction of the thermal and the mechanical strain, represents only the strain induced by the interstitial deuterium. (c) The amount of deuterium in the matrix solid solution evaluated using the caxis strain and Eqn. (2). . . . . . . . . . . . . . . . . . . . . . 


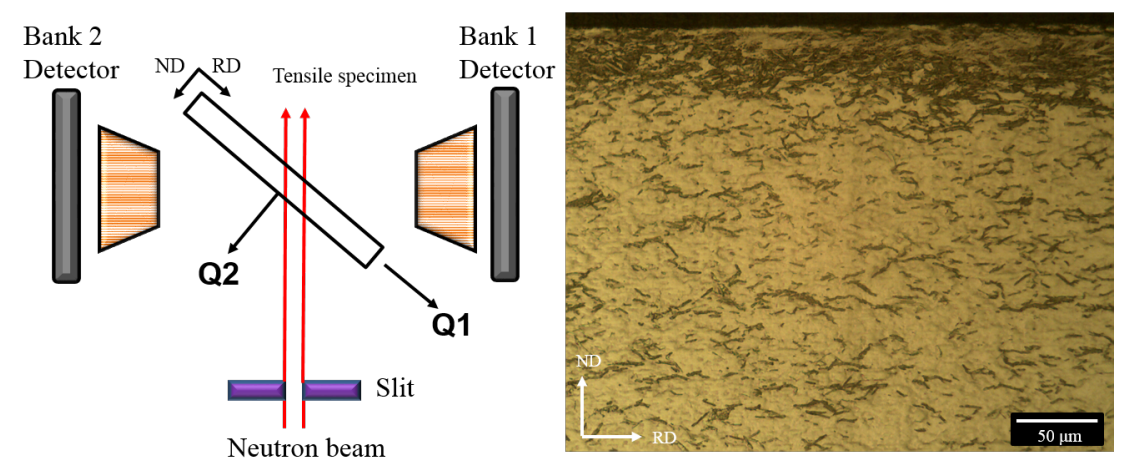

(a)

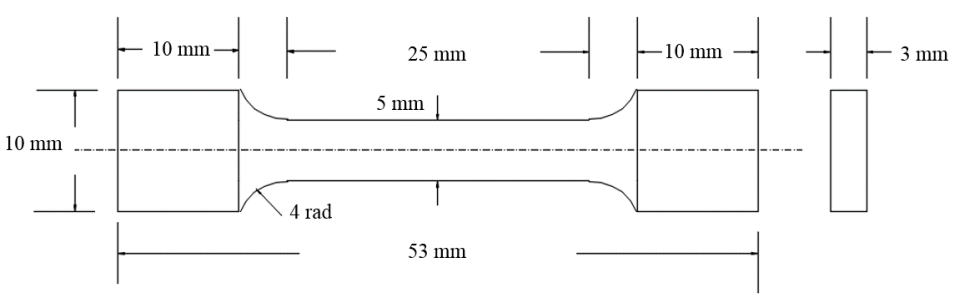

(b)

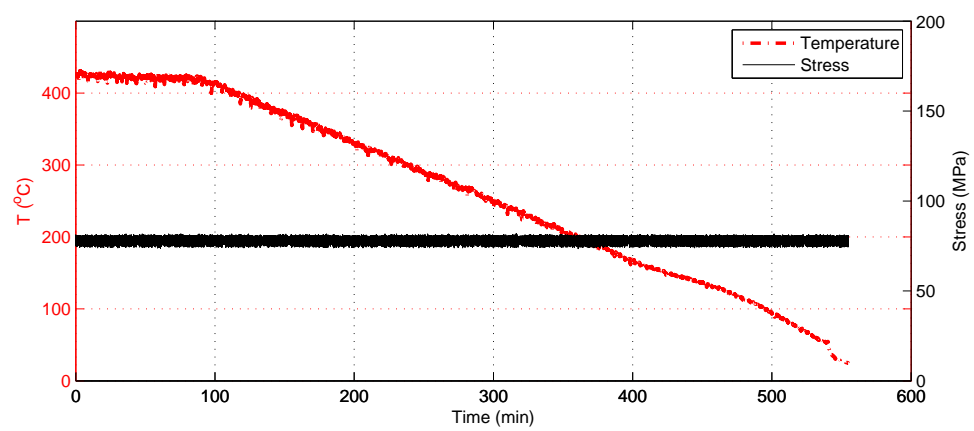

(c)

Figure 1: (a) Experimental arrangement of VULCAN. Bank 1 and Bank 2 recorded the lattice planes with their normal parallel to Q1 and Q2, respectively. Q1 and Q2 correspond to the RD and ND in terms of the sample orientation, respectively. The external load is applied along the RD, while the deuteride rim/blister is on the ND surface. The incident neutron beam is at $45^{\circ}$ with respect to the ND. (b) The dimension of the dog-bone tensile specimen. (c) The thermal and mechanical history throughout the in situ experiment. 


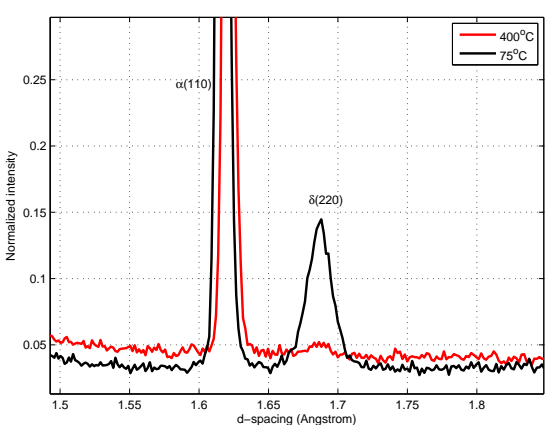

(a)

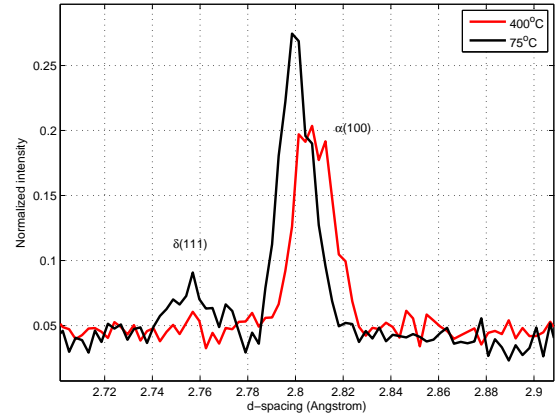

(b)

Figure 2: Examples of partial neutron diffraction spectrums from the (a) $\delta(220)$ reflection and the (b) $\delta(111)$ reflection. The red solid line represents the spectrum taken at $400{ }^{\circ} \mathrm{C}$ while the black solid line was taken at $70{ }^{\circ} \mathrm{C}$. 


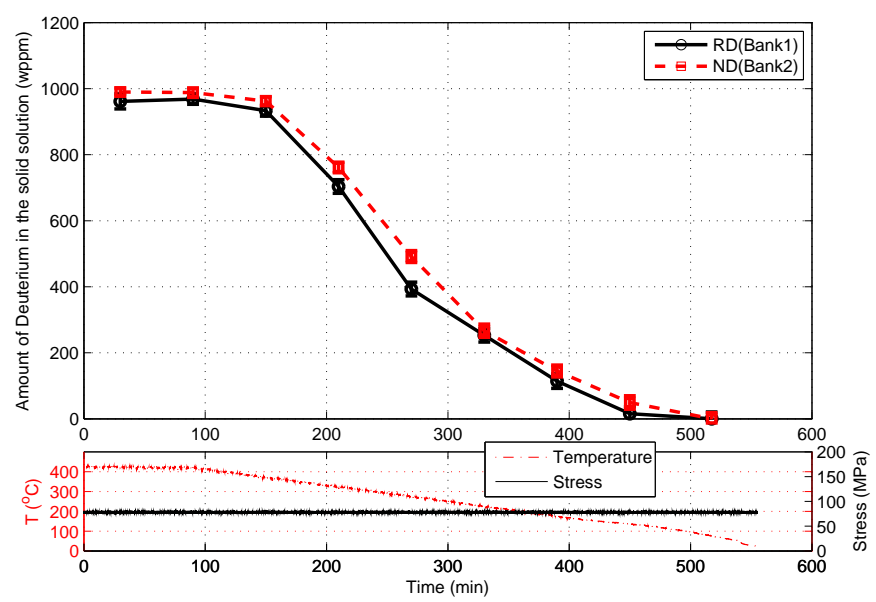

(a)

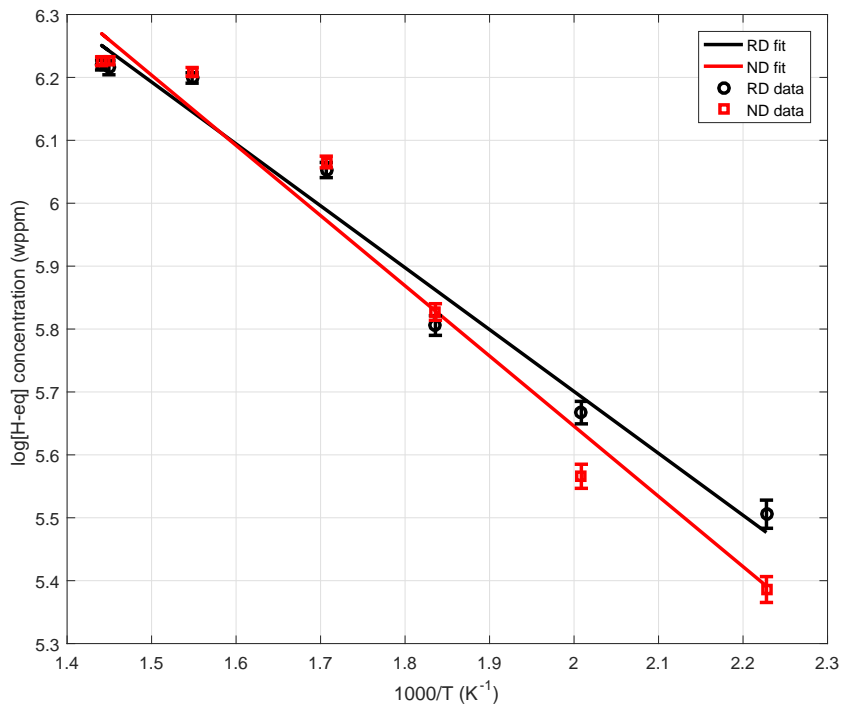

(b)

Figure 3: (a) Evolution of the deuterium concentration in the zirconium solid solution in the unit of wppm. The uncertainties are within the size of the data points. A subplot shows the corresponding temperature and stress at each data points. (b) Arrhenius fit on the evolution of deuterium concentration in the zirconium solid solution. 


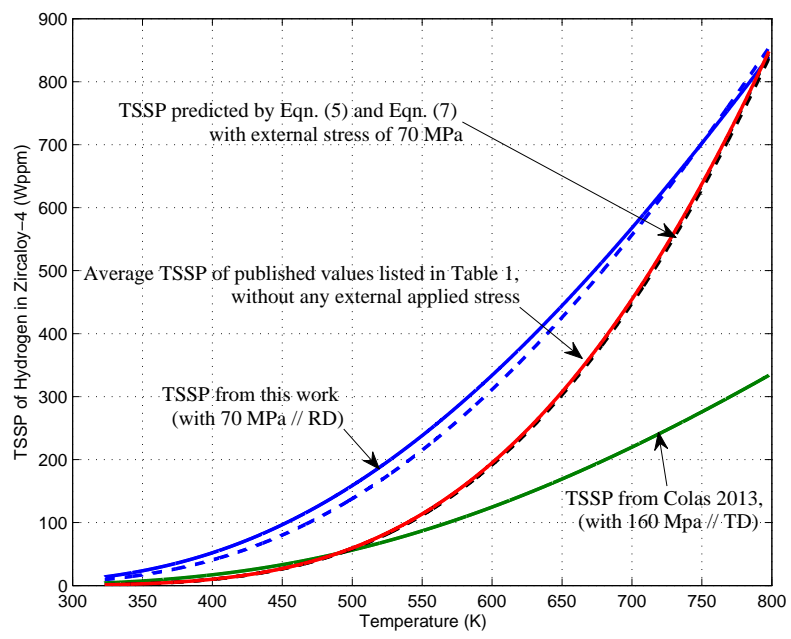

(a)

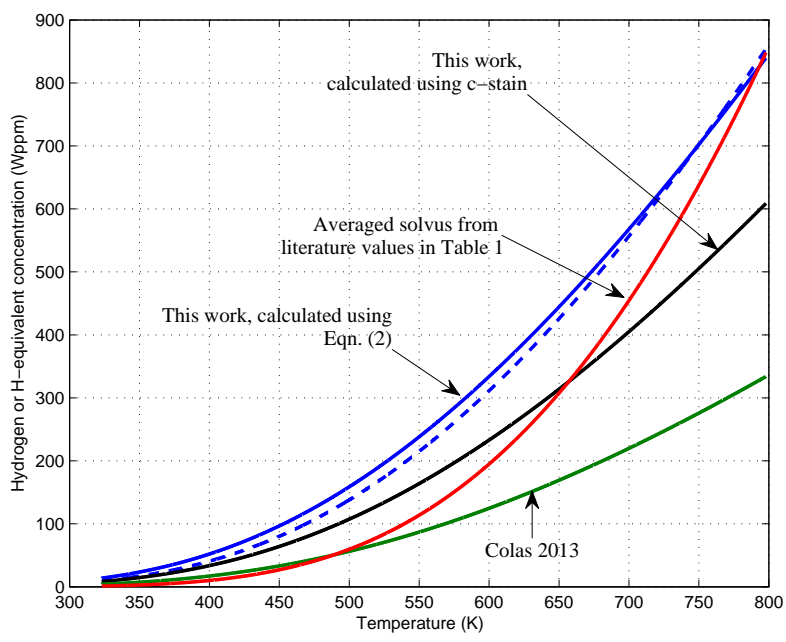

(b)

Figure 4: (a) TSSP of hydrogen in the Zry-4 solid solution, showing the discrepancy between the experimental observation and theoretical calculation for the effect of the external stress on the solvus. Blue lines represent the experimental measurements in this work along the two directions with $78 \mathrm{MPa}$ of tensile stress applied along the RD. Red line is an average solvus of several literature values that are listed in Table. 1, also serves as the zero external stress solvus for the theoretical models (Eqn. (6) and (7)). Black line is a theoretically calculated solvus based on the red line with a $78 \mathrm{MPa}$ uniaxial stress applied. (b) Same as (a) but including the solvus calculated using the c-axis dilatation in this work represented as a black line and excluding the theoretically calculated solvus for clearance . 

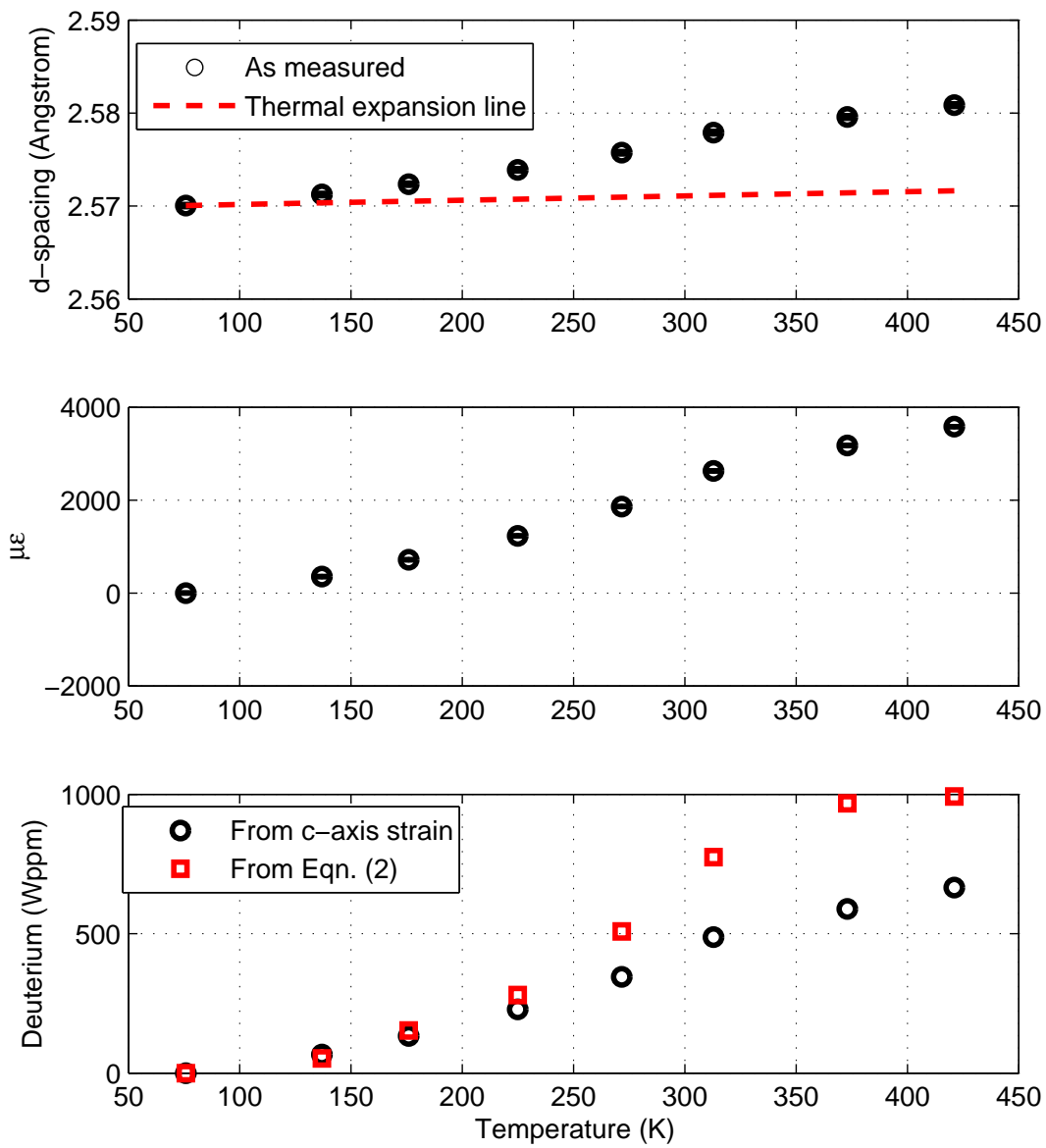

Figure 5: (a) The evolution of the $c$ lattice parameter as function of the temperature in this study along with the predicted thermal expansion line displayed in a red dash line. (b) The strain on the c-axis after subtraction of the thermal and the mechanical strain, represents only the strain induced by the interstitial deuterium. (c) The amount of deuterium in the matrix solid solution evaluated using the c-axis strain and Eqn. (2). 